construction de fosses auprès d'écluses étanches. En raison des difficultés actuelles, ce crabe pourrait être utilisé frais pour l'alimentation humaine et en poudre pour la nutrition des animaux:

\title{
BIBLIOGRAPHIE
}

Hoestzandt (H.), 1940. - L'Eriocheir sinensis dans le Nord de la France (19371939). - Bulletin Français de Pisciculture, no 121, pp. 101-125.

Kamps (L. F.), 1937. - De Chineesche Wolhandkrab in Nederland, 108 pagés.

Leloup (E.), 1938. - La propagation dù Crabe chinois en Belgique pendant l'année 1937. - Bulletin du Musée Royal d'Histoire Naturelle de Belgique, XIV, no 14, 25 pages.

Lestage (J. A.), 1939. - Nouvelles recherches sur l'extension en Belgique du Crabe chinois. - Annales de la Société Royale Zoologique de Belgique, LXX,

pp. 5-8.
LESTAGE (J. A.), 1941. - L'extension du Crabe chinois en Belgique. - Péche et Pisciculture, Septembre-Octobre ${ }^{-1941}$, pp. 118-119.

Peters (N.) und Hoppe (H.), 1938. - Uber Bekaempfung und Verwertung der Wollhandkrabbe. - Mitt. Hamb. Zool. Mus. Bd. 47, pp. 140-171.

Peters (N.) und Pannina (A.), 1933. - Die Chinesische Wollhandkrabbe in Deutschland. - Zoologischer Arizeiger; Bd. 104, 180 pages.

\section{NOTES ET ESSAIS \\ CONCERNANT LE NOURRISSAGE DE LA TRUITE}

\author{
Par le Comte Victor D'ANSEMBourg, \\ Pisciculteur à Assenois (Luxembourg belge).
}

Les expériences dont il est question içi pêchent par plus d'un côté : leur durée est parfois trop courte, le nombre des individus mis à l'essai est par trop restreint, dans certains cas, etc. Les premières d'entre elles ne sont pas de date fort récente et se situent à une époque où nous débutions dans l'art du nourrissage. Elles n'avaient d'autre but que de nous donner des lumières personnelles sur l'un ou l'autre détail qui nous paraissait obscur ou discutable, notamment sur la valeur pratique de certains alíments. Mais, pensant qu'il est peut-être bon de verser le plus de pièces possible au dossier de l'alimentation de la Truite, mểme si elles ont de gros défauts et si ce sujet ne semble guère comporter de réalisations pratiques actuelles pour la plupart d'entre nous, nous livions ces quelques essais, non comme des modèles d'expérimentation, ni comme des exemples de granids rendements, màis simplement pour ce qu'ils valent.

L'exposé et la critique dę expériences seront suivis d'une tentative de détermination de la ration d'entretien et de commentaires sur cette question.

Article available at http://www.kmae-journal.org or http://dx.doi.org/10.1051/kmae:1942008 
Les expériences furent exécutées dans les Ardennes belges. Le sol y est généralement schisteúx et les eaux y offrent des températures et des débits à variations très étendues. Cette région, somme toute, se prête fort bien à la culture extensive des étangs à Truites et à l'élevage naturel des alevins, et un peu moins bien au nourrissage intensif. Les eaux étant particulièrement froides en hiver, et nos essais ayant été pratiqués en dehors de l'été, nous ne verrons guère d'accroissements intéressants. Ce n'était d'ailleurs pas le but poursuivi, car, sauf dans le premier cas, il s'agissait d'6preuves comparatives.

Les données concernant la ration d'entretien ont été fournies par une station différente, offrant des eaux à débit volumineux, régulier et à températures moins variables, toutes conditions propices au nourrissage et à l'exploitation intensive.

\section{I. - EssAIs DIVERs}

Notons au préalable que la ration journalière proportionnelle au poids des poissons (R. P., pour abréger) est calculée chaque fois d'áprès le poids total moyen des Truites (poids initial plus poids final divisé par deux). Elle se base sur le nombre total des jours de l'expérience ou de la période considérée, et non sur le nombre des distributions de nourriture, qui est ici - et cela à cause de la température basse qui a présidé à nos essais inférieur au nombre de jours.

Quant au quotient alimentaire (Q.A.), c'est-à-dire le nombre de kilos de nourriture produisant up kilo de chair de poisson, il n'y a pas lieu de faire de distinction entre le Q.A. relatif et le Q.A. absolu (1), car la mortalité et les fuites furent nulles, le total exact des sujets mis à l'épreuve ayant été retrouvé chaque fois.

Popr l'expoé des données et des résultats, nous nous inspirons de lạ mise en tableaux adoptée par H. Barthélemy et E. Musard dans leur intéressante étude parue ici-même en 1937 (2). Toutefois, notre façon de calculer la ration próportionnelle diffère de la leur.

Ajoutons que les bassins qui ont servi à ces expériences étaient tous ciméntés, et que les sujets étaient, dans chaque cas, des truitelles arc-enciel.

(1) Le Q.A. relatif indique le rendement global en Truites vivantes compare à la quantitê totale de nourriture employée. Le $Q . A$. absolu tient compte de la nourriture perdue par le fait des animaux morts ou échappés ainsi que du poíds initial de ces animaux. Les quotients alimentaires mentionnés par les auteurs sont presque toujours des Q.A. relatifs. Pour le trutticulteur qui désire apprécier le résultat économique d'une période de nourrissage dans l'ensemble de son établissement, c'est le Q.A. relatif qui compte, les Truites mortes ou échappées étant pratiquement perdues. Mais, pour l'experrimentation scientiflque, pour la mise à l'essai d'un aliment, c'est le Q.A. absolu qui devrait toujours être calculé.

(2) Voir Bulletin. - Avril 1937 no 106, p. 241. - Mai 1937 no 107, p. 265. Juin 1937 no 108, p. 289. 
Essar N*1. - Lañt caillé.

Le lait caillé, utilisé parfois dans le nourrissage des alevins, les jours où la rate se trouve en quantités insuffisantes, est-il vraiment profitable aux jeunes Truítes ? Nous voulions en avoir le cour net. Poir cela il fallait tenter d'en déterminer le Q.A. Les alevins prennent fort bien cette nourriture qui se réduit facilement en unè multitude de parçelles appropriées aux dimensions de leur bouche. La gránde visibibilité de ces flocons blancs, qui doivent produire dans le monde aquatique l'effet d'une chute lente de neige, attire tous les élèves, dońt les estomacs, bien vite bourrés, deviennent blancs a leur tour et laissent transparaitre cette couleur au dehors. Cela se rèmarque surtout chez les alevins de Truite fario.

Notre essai fut fait, sans appoint d'autre aliment, sur des petites truitelles, celles-ci, a défaut d'instruments de grande précision, étant plus faciles à peser que des alévins.

TABleau I

Durée de l'expérience : 1 mois (22 Mars au 29 Avril 1935).

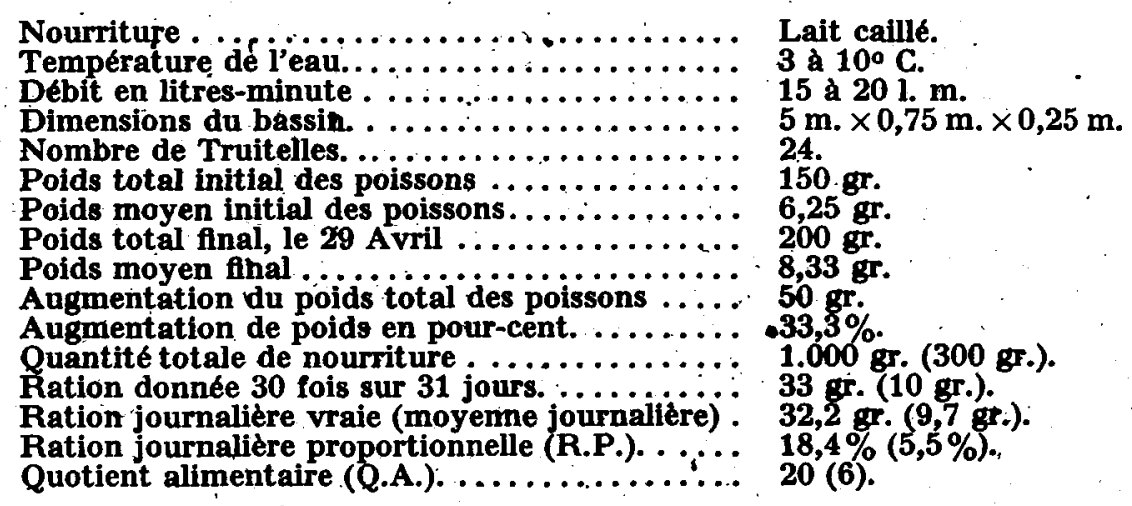

La quantité dé fromage blanc (lait caillé égoutté) correspondant à un kilo de lait caillé étant d'environ 300 grammes, nous avons indiqué entre parenthèses les chiffres qu'aurait théoriquement donnés le fromage égoutté. Le Q.A. de celui-ci serait environ égal à 6 (ou un rien plus élevé, pour ne pas oublier le sérum ou petit lait qui est éliminé quand le lait caillé s'égoutte).

Cette expérience donnerait à croire qu'une alimentation exclusive au lait caille ou au fromage blane peut satisfaire les exigences des Truitelles. Mais nous savons que cet aliment est incomplet; que, malgré la haute teneur en protéine brute indiquée par les tables de la compos'tion nutritive, il ne contient pas tous les éléments de croissance de la protéine, et qu'il est très pauvre en fer. Les auteurs affirment que le nourrissage au fromage blanc pur provoque I'anémie à brève échéance. D'après eux, le Q.A. est compris entre 10 et 15. 
Si nous arrivons à un Q.A. d'environ 6, nous devons toutefois faire la restriction suivante. L'espace donné à un trop petit nombre de sujets et le fait que l'eau employée était une eau de ruisseau laissent supposer que les Truitelles ont peut-être bénéficié d'un surcrott de nourriture naturelle, infinitésimal, il est vrai, car l'eau était tamisée et le bassin cimenté. Ces apports de faune nutritive constituent une cause d'erreur qu'il n'est pas toujours facile d'éviter, mais que l'on devrait réduire au minimum, en employant de l'eau de source canalisée et en parquant un grand nombre de sujets à l'étroit. Négligeable dans nos essais ultérieurs, qui ont surtout un but comparatif, cette possibilité d'erreur laisse un doute sur l'exactitude rigoureuse de nos chiffres, notamment de celui du Q.A.

Ce résultat nous a cependant donné l'assurance que le lait caillé fournit une augmentation de poids et qu'il peut jouer un rôle honorable dans l'alimentation des jeunes Truites; mais il ressort de l'examen de sa composition chimique qu'il doit être complété par d'autres principes nutritifs.

Quant à son emploi dans le premier élevage des alevins, l'usage que nous en avons fait en dehors de cet essai, en l'ajoutant accessoirement à la rate, n'a pas donné de mauvais résultats. Il est toutefois fort possible que, là où la rate est abondante, celle-ci, donnée seule, procure un meilleur Q.A. que le mélange, ou bien que le nourrissage à la rate, entrecoupé de distributions de lait caillé. C'est une expérience à faire. Mais ce qui réussit encore mieux à l'alevin, c'est l'élevage en extensive, sans aucun nourrissage, basé uniquement sur l'alimentation rigoureusement complète que lui offre la faune nutritive. Pour notre part, nous n'en faisons plus d'autre, du moins en ce qui concerne les alevins de fario.

\section{Essai No 2:- Crevettes séchées.}

Deux lots de Truitelles furtent soumis à des conditions de milieu identiques. Seule la nourriture différait : d'un côté, la ration comportait une certaine quantité de Crevettes séchées, - de l'autre cốté, la ration était ordinaire.

En raison de la température presque glaciale de đ'eau, les Truites ne sont pas très bien venues à la nourniture, surtout du 14 Janvier au 15. Février. Elles cueillaient peu de morceaux à la surface de l'eau, ramassant plutôt ceux qui tombaient au fond du bassin, et il so peut fort bien qu'une petite partie des rations n'ait pas été consommée.

Pourtant, - '’augmentation de poids fut très nette, quoique modeste, pendant ce premier mois, et négative ou à peu près nulle du 15 Février au 11 Mars. On voit que, durant cette deuxième période, les besoins d'entretien n'étaient pas couverts dans le bassin $n^{\circ} 2$, puisque le poids y a subi une légère régression, tandis que, dans le bassin $n^{\circ} 1$, une ration proportionnelle à peine plus élevée a fait augmenter, bien que très faibłement, le poids général. La cause de ce flottement de la croissance, après le 15 Février. 
est peut-être à rechercher, tout simplement, dans le fait d'une légère reprise saisonnière de la température amenant une recrudescence parallèle dés exigences alimentaires, pour lesquelles une R.P. demeurée stationnaire devenait insuffisante.

Tableau II

Durée de l'expérience : 57 jours (14 Janvier au 11 Mars 1936).

\begin{tabular}{|c|c|c|}
\hline & Bassin 1 & Bassin 2 \\
\hline 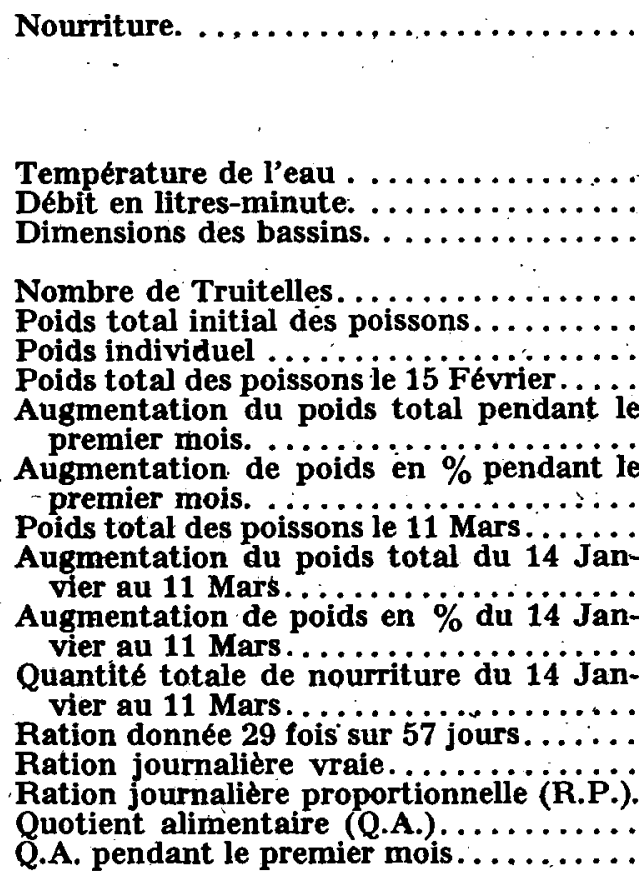 & $\begin{array}{l}15 \text { gr. de Crevettes } \\
\text { cuites. } \\
15 \text { gr. de viande et } \\
\text { abats cuits } \\
5 \text { gr. de son de blé. } \\
1 \text { a } 50 \mathrm{C} . \\
15 \text { a } 20 \text { l. m. } \\
\text { Comme dans l'es- } \\
\text { sai no } 1 . \\
20 \\
790 \mathrm{gr} . \\
39,5 \mathrm{gr} . \\
870 \mathrm{gr} . \\
80 \mathrm{gr} . \\
10,1 \% \\
880 \mathrm{gr} . \\
90 \mathrm{gr} . \\
11,4 \% \\
1.015 \mathrm{gr} . \\
\mathbf{3 5} \mathrm{gr} . \\
17,8 \mathrm{gr} . \\
\mathbf{2 , 1} \% \\
\mathbf{1 1} \\
\mathbf{6 , 3}\end{array}$ &  \\
\hline
\end{tabular}

Le résultat du lot nourri en partie avec des Crevettes paralt meilleur que celui du bassin $n^{\circ} 2$, et cela pour les deux périodes. On pourrait objecter que, si les rations des deux lots étaient égales en poids, elles ne l'étaient pas en unités nutritives. Le poids indiqué pour les Crevettes étant celui du produit " sec " et, par conséquent, à protéine concentrée, le premier bassin a da recevoir plus de protéine que le deuxième. Il devait donc nécessairement fournir un Q.A. plus favorable, si, bien entendu, ces Crevettes étaient de bonne qualité.

Nous reparlerons de la teneur en protéine des Crevettes séchées dans la critique de l'essai suivant. Notons simplement que, si. l'on peut faire état des indications de certains auteurs qui assignent $34,6 \%$ de protéine brute aux Crevettes séchées et $20 \%$ en moyenne à la viande, et si nous ajoutons que, par le fait de la cuísson, la viande avait aussi subi une cer-. 
taine concentration de ses principes azotés, nous ne pouvons conclure à une différence bien marquée entre les quantités de protéine contenues dans les deux rations. La teneur en protéine de la viande cuite était probablement d'au moins $30 \%$; quant aux Crevettes, la grande quạtité de chitine inassimilable qu'elles renferment est généralement comptée dans la protéine brute. Les deux lots de Truitelles ont donc vraisemblablement bénéficié de quantités sensiblement égales de protéine.

L'action des Crevettes nous paraissant, somme toute, favorable, cet aliment fut repris l'année suivante dans un autre essai, qui confirma sa valeur nutritive.

Essar $\mathrm{N}^{\circ}$ 3. - Crevettes séchées et farine de poissons.

Cette expérience, qui fut de courte durée, les bassins étant requis par d'autres' peuplements, fut faite à titre de comparaison entre les Crevettes séchées et une farine de poissons qui est vraisemblement la même que. la farine C. expérimentée par Barthelemy et Musard (loc. cit.).

Tableav III

Durée de l'expérience : 17 jours (12 au 29 Avril 1937).

\begin{tabular}{|c|c|c|}
\hline$\therefore$ & Bassin 1 & Bassin 2 \\
\hline 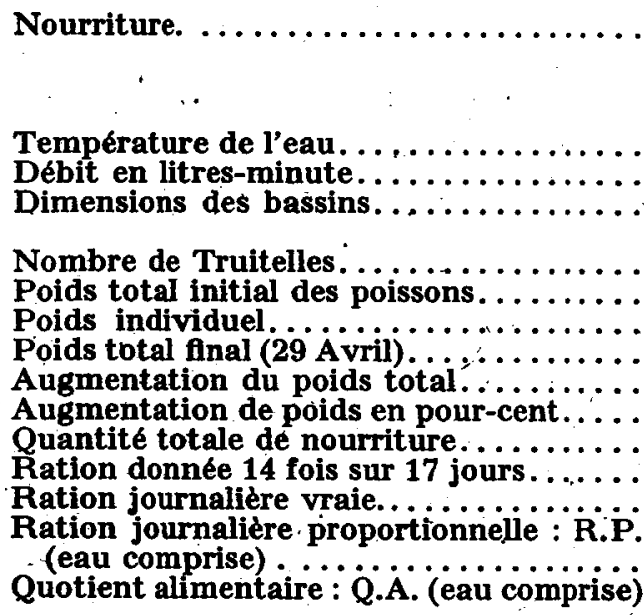 & $\begin{array}{l}\text { Farine de pois- } \\
\text { sons + eau. } \\
\text { Viande et abats } \\
\text { cuits. } \\
6,5 \text { a } 7,50 \mathrm{C} . \\
60 \text { a } 100 \text { l.-m. } \\
7,70 \times 1,35 \\
\times 0,55 \mathrm{~m} . \\
200 \\
2.700 \mathrm{gr} . \\
13,5 \mathrm{gr} . \\
2.960 \mathrm{gr} . \\
250 \mathrm{gr} . \\
\mathbf{9 , 2} \% \\
1.680 \mathrm{gr} . \\
120 \mathrm{gr} . \\
99 \mathrm{gr} . \\
3,5 \% \\
6,72\end{array}$ & 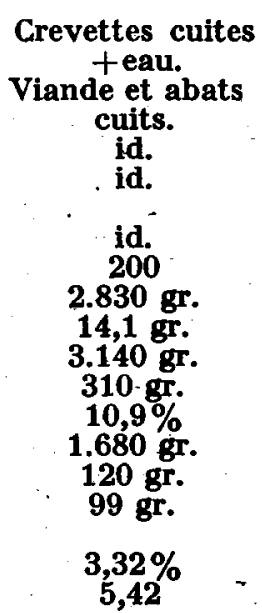 \\
\hline
\end{tabular}

Les chiffres du tableau III révèlent un rendement plus favorable dans le bassin $n^{\circ} 2$ (Crevettes séchées) que dans le bassin $n^{\circ} 1$ (farine de poissons), et cela malgré une R.P. un peu plus faible et malgré le fait que les Crevettes étaient cuites.

La ration comportait, outre 60 grammes de viande et d'abats dans les deux cas : 20 grammes de farine de poissons additionnée de 40 grammes 
d'eau, soit 60 grammes de pâte, d'une part, et 60 grammes d'un mélange de Crevettes et d'eau, d'autre part. La quantité d'eau ajoutée aux Crevettes fut réglée par le pouvoir d'abeorption de celles-ci : 1.800 grammes de produit sec donnant 3 kilogrammes de Grevettes cuites imbibées d'eau, le mélange de 60 grammes contenait 36 grammes de Cirevettes séehées.

La cuisson, en rendant feur protéine moins assimilable, désavantageait les Crevettes au profit de la farine de poissons qui était demeurée à l'état cru. Mais ces Crevettes séchées sont accompagnées de pinces de Crabes et d'autres matières dures qui se ramollissent difficilement si elles ne sont pas cuites.

La ration du bassin $n^{\circ} 2$ comportait plus de matière sèche que celle du bassin $n^{\circ} 1$ (pour autant que la matière fút complètement sèche dans les deux cas). Cette différence est égale à 36 grammes de Crevettes moins 20 gràmmes de farine de poissons, soit 16 grammes multipliés par 14 jours $=224$ grammes.

Supposons que les deux produits secs contiennent à poids égal la même dose de protéine digestible: En ce cas, l'excédent du poids sec des Crevettes amènerait un surcroft proportionnel de protéine en faveur de la ration du bassin $n^{\circ} 2$. Supprimons les poids d'eau incomporés dans les rations de 120 grammes et nous trouvons que le bassin $n^{\circ} 1$ a reçu : $80 \times 14=$ 1.120 grammes de nourriture, le bassin $n^{\circ} 2: 96 \times 14=1.344$ grammes. Le Q.A. s'établit ainsi à 4,48 pour la farine de poissons et à. 4,33 pour les Crevettes.

Dönc, même en tablant sur la marchandise considérée à l'état sec, le Q.A. reste un peu meilleur pour les Crevettes que pour la farine en question, bien que le prix de celle-ci, basé sur la forte concentration de protéine et sur le poids que doit faire la pâte obtenue par addition de l'eau, soit, rendu ici, 3,5 fois plus élevé que celui des Crevettes séchées.

Mais il est probable que la teneur en protéine digestible est, du moins théoriquement, plus élevée pour la farine de poissons que pour les Crevettes séchées. La facture du fournisseur de ces dernières indique bien $55 \%$ d'albumine, mais qu'y a-t-il de digestible dans cette albumine, qui comprend certainenenent la chitine des carapaces, fortement représentées dans ce produit? Les tableaux de la composition chimique des aliments assignent à la farine maigre de poissons $52,5 \%$ de protéine brute, et aux Crevettes séchées $34,6 \%$. Pour autant que ces chiffres puissent s' appliquer au cas présent, - et en tout cas, ils nous paraissent plus rẹprésentatifs de là teneur en principes utilisables de ces aliments et ils possèdent une valeur de comparaison, - il n'est pas interdit de supposer qu'en donnant, d'une part, 20 grammes, et, d'autre part, 36 grammes de matière sèche, nous donnions en réalité des quantités de protéine brute à peu près équivalentes.

Il faut ajouter que, la farine de poisson ayant une forte tendance à se dissocier dans l'eau, il s'en est perdu peut-être un peu plus qu'il ne se perdait de Crevettes, et, cela, malgré les précautions prises. Nous ne conclurons donc pas, d'une façon absolue, qu'un produit est meilleur que 
l'autre, mais nous dirons que, dans les conditions de notre expérience, les Crevettes ont donné un meilleur rendement.

Les Grevettes séchées, même si elles sont cuites, constituent donc un excellent appoint à la nourriture (elles ont, en outre, l'avantage de corriger e mauvais goût que pourraient donner à la Truite certains régimes alimentaires). Quant à la farine en question, si l'on peut lui reprocher son prix beaucoup trop élevé et la difficulté d'en faire une pâte consistante, il ne semble pas qu'on puisse lui dénier ses qualités nutritives, sa pureté, sa fraîcheur, ainsi que l'absence de matières échauffantes et de toxines.

Essai $N^{\circ} 4 .-$ Croissance de Truites de même taille et d'áges différents.

Il s'agit ici d'une expérience d'un autre ordre, dont le but n'est plus de mettre un aliment à l'épreuve, mais les poissons eux-mêmes.

Ayant; cette année-là, un pourcentage, d'ailleurs infime, de sujets de deux étés présentant un retard extraordinaire de croissance, leur comparaison avec des Truitelles normales nous parut intéressante à tenter, au point de vue de leur augmentation de poids, dans des conditions identiques de milieu et de nourriture.

Un " choix" fut fait parmi les plus petits de ces spécimens minuscules, et il se trouva ainsi 120 Truites âgées d'un an et demi faisant au total le même poids que 128 Truitelles âgées de 5 mois.

La sélection méthodique des reproducteurs réduit, et exclut même, les chancés de voir des sujets de deuxième année aussi chétifs; mais ce cas peut se présenter si l'origine de ceux-ci remonte à des œufs de provenapce extérieure, nés de parents inconnus, comme cela arrive si l'on désire compléter, par des achats, la production des géniteurs élevés et triés sur place; et si à cette cause première s'ajoute une alimentation insuffisante des Truitelles d'un été de la plus petite catégorie.

Les deux lots de Truites reçurent des rations identiques de 70 grammes, comprenant, soit de la rate pure (donnée treize fois), soit un mélange de viande, abats, Crevettes ou farine de poissons et remoulage (donné dix fois) 
Durée de l'expérience : 38 jours (27 Octobre au 4 Décembre 1938).

\begin{tabular}{|c|c|c|}
\hline$\therefore$ & Bassin 1 & Bassin 2 \\
\hline 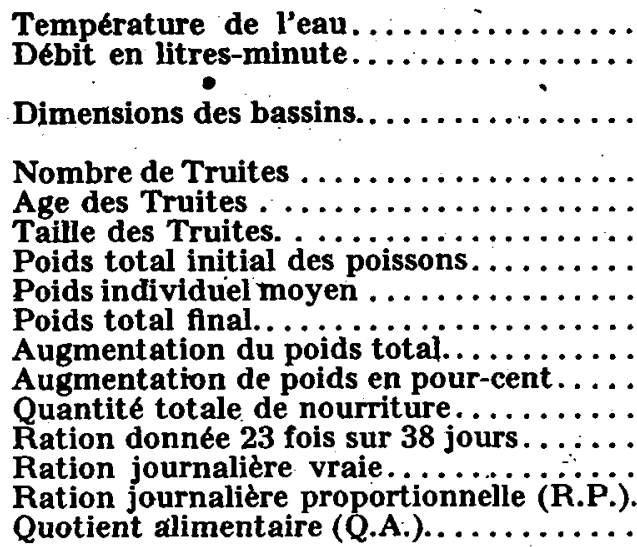 & $\begin{array}{c}2,5 \text { à } 6 \circ \mathrm{C} \\
\text { Comme dans l'es- } \\
\text { sai no } 3 . \\
\text { Comme dans l'es- } \\
\text { sai no } 3 \\
128 \\
5-6 \text { mois } \\
8-11 \mathrm{~cm} . \\
1.300 \mathrm{gr} . \\
10,1 \mathrm{gr} . \\
1.560 \mathrm{gr} . \\
260 \mathrm{gr} . \\
20 \% \\
1.610 \mathrm{gr} . \\
70 \mathrm{gr} . \\
42,4 \mathrm{gr} . \\
2,96 \% \\
6,2\end{array}$ & $\begin{array}{c}\text { id. } \\
\text { id. } \\
\text { id. } \\
120 \\
\text { un an et demi. } \\
7-13 \mathrm{~cm} . \\
1.300 \mathrm{gr} . \\
10,8 \mathrm{gr} . \\
1.585 \mathrm{gr} . \\
285 \mathrm{gr} . \\
21,9 \% \\
1: 610 \mathrm{gr} . \\
70 \mathrm{gr} . \\
42,4 \mathrm{gr} . \\
2,94 \% \\
5,6\end{array}$ \\
\hline
\end{tabular}

Ce résultat peut paraître surprenant, car les sujets arriérés ont mauvaise réputation, et l'on voit qu'ici ils se sont comportés aussi bien, pour ne pas dire mieux, que les sujets normaux.

Il est toujours bon de rechercher les 'causes d'erreur qui s'insinuent si facilement dans les expériences. En principe, les poissons doivent être à jeun au moment des pesées. Sinon; il faudrait au moins que les tubes digestifs des sujets à comparer fussent indentiquement chargés. Dans le cas présent, les truitelles mises dans le bassin $n^{\circ} 1$ venaient, avant l'essai, mieux à la nourriture que celles qui étaient destinées au bassin $n^{\circ} 2$. Il n'est donc pas impossible que les sujets normaux se soient trouvés, au moment de la première peșée, moins à jeun que les sujets arriérés.

Voyons comment cette différence initiale hypothétique aurait pu modifier nos chiffres, et admettons que le contenu stomacal du lot $n^{\circ} 1$ pesait au total 40 grammes de plus que celui du lot $n^{\circ} 2$, cette différence représentant $3 \%$ du poids des Truites du lat $n^{\circ} 1$.

Le poids initial de celles-ci, supposées à jeun ou nourries au même degré que les autres, se réduit à $1.300-40=1.260$ grammes, et l'augmentation de poids passe à 300 grammes. Le Q.A., basé sur une quantité totale de nourriture de $1.610+40$ grammes, s'établit à 5,5 contre 5,6 pour fe lot $n^{\circ} 2$. Cette supposition trìs large ne modifie pas nos résultats jusqu'à révéler une nette sưpériorité des sujets plus jeunes, elle amène simplement l'équivalence entre les rendements des deux bassins.

Dans une population de poissons restés petits, il y a lieu de distinguer les individus rétifs des sujets bien disposés : ceux qui ont un mauvais facteur de croissance et ceux dont la cause du retard est extérieure, non héré- 
ditaire, non individuelle et qui possèdent une bonne virtualité d'accroissement. Dans le cas présent, ces deux tendances étaient vraisemblablement représentées, et ce faìt fut confirmé pàr l'accentuation de la divergence des tailles, déjà très diverses au début de l'essai.'

Certains " gros ", mais aussi certains " petits ", devaient donc appartenir à la classe des retardataires pleins de bonnes dispositions. Tout trutticulteur aura sans doute remarqué, après ses triages, qu'un certain nombre de sujets d'une catégorie de taille inférieure sortent brusquepent du rang et se mettent à dépasser les plus petits de la catégorie supérieure. Il n'y a là rien d'étonnant, car ceux-là sont de bons sujets retardés, et ceux-ci de mauvais sujets avancés. Mais ce qu'il faut noter en plus ici, c'est que cette croissance des individus les mieux doués parmi les déshérités du bassin $\mathrm{n}^{\circ} 2$ a été telle, que la marche du poids total s'en est trouvée au moins aussi bonne que celle du bassin $n^{\circ} 1$.

Nous ne conclurons donc évidemment pas que chacun des sujets malingries a gagné autant de poids que chacun des sujets normaux. Il y a des cas de rabougrissement incurable. Il n'en reste pas moins que, dans une population de Truites demeurées petites, il se trouvera, en général et malgré tout, d'intéressantes possibilités de croissance, grấce à un nombre plus considérable qu'on ne croit d'habitude, de bons sujets qui se manifestent tout à coup, ou avec une soudaineté apparente. Il faudrait isoler ceux-ci au fur et à mesure de leur apparition, pour supprimer, en fin de compté, les individus chétifs qui ne donnent pas d'espoir de les voir atteindre 100 à 150 grammes dans un délai acceptable. Dans notre cas, les sujets arriérés avaient été plutôt délaissés et sous-alimentés ; mais il est certain qu'un bon nourrissage et des triages répétés auraient permis d'en faire pousser, en deuxième année, à peu près convenablement la majeure partie.

Il ne nous paraft donc pas utile de trop se hâter de supprimer, pour la raison qu'ils constituent un poids mort dans l'exploitation,' les individus malingres ou considérés comme tels. Il semble préférable de surseoir à cette exécution pour laisser une sélection s'établir naturellement et peu à peu entre eux. Ceci nécessite, bien entendu, des triages très fréquents.

\section{II. - Ration d'entretien}

\section{1. - Essai de détermination de la ration d'entretien.}

Il ne s'agit pas d'une expérience dirigée dans ce sens, mais plutôt de déductions tirées de l'examen de divers résultats. L'essai ne serait pas difficile à conduire expérimentalement, et nous nous proposons de le faire plus tard. Malgré son importance, cette question a été relativement peu étudiée pour la Truite.'

Et, pourtant, il y aurait grand intérêt à délimiter, dans des conditions déterminées de température, d'âge, de taille, d'espèce, etc., d'une part, la tranche improductive de la ration, c'est-à-dire celle qui se transforme en 
énergie, en chaleur, en graisse, et se charge ainsi de l'entretien de la vie, et, d'autre part, sa tranche productive, celle qui fournit les matériaux de construction du corps et assure ainsi la cmoissance.

La question, en d'autres mots, est de chercher à connaftre le nombre de kilos dé nourriture nécessaires chaque jour pour maintenir en poids une population de cent kilos, le surplus seul réalisant l'accroissement de. ce poids. La tranche improductive est ce que l'on appelle la ration dientretien (R.E.). Il faut bien noter que celle-ci a toujours la priorité sur la ration productive, et qu'il n'y aura jamais de croissance tant que les besoins énergétiques ne sont pas couverts.

Barthélém et Musard (1) estiment dans un cas particulier la R.E. à $7,5 \%$ environ. La R.P. de 7,81 qui sert de base à cette déduction est calculée en fonction du poids initial des poissons et non d'après leur poids moyen pendant la période considérée; ensuite, les jours de jeane sont déduits. Si nous faisons le calcul en suivant la règle proposée au début de cet article, nous arrivons à une R.P. de 6,57 et à une R.E. approximative de 6. Ce chiffre dépasse cependant encore ceux que nous avons trouvés. Peut-être une partie de la nourriture a-t-elle teté refusée par les Truites, comme ce fut probablement le cas dans notre essai $n^{\circ} 2$.

Nous avons vu, dans les expériences oi-dessus, que des R.P. de 3,3\% (tableau III) et de 2,9\% (tableau IV) ont fourni une augmentation de poids, c'est-à-dire un excédent utilisé pour la croissance. Remarquons en passant que le poids des rations comprenant des produits secs y est'compté avec l'eau réstituée d̀ ceux-ci. .

Nous ne tirenons aucune conclusion de la comparaison des R.P. : $2,1 \%$ et $2,02 \%$ (tableau II), qui amenèrent, la première, un accroissement, la deuxième, une diminution de poids, car les rations étaient de composition différente ; la protéine était trop concentrée et, de plus, il n'est pas certain que toute la nourriture ait été consommée.'

Lors de nos débuts dans le nourrissage de la Truate, on nous avait affirmé qu'une Truite doit recevoir chaque jour, en nourriture, $5 \%$ de son poids en été, et $2 . \%$ en híver. Ces chiffres sont, bien entendu, insuffisants dans de nombreux cas, et ils n'ont pas la souplesse que requièrent les variations thermiques, les différences de taille et d'autres facteurs. Si nous citons ce souvenir, c'est pour constater que ces R.P. nous ont généralement donné une augmentation de poids là où nous les avons appliquées ; en d'autres mots, la R.E. se situait en dessous de ces chiffres.

En nous appuyant sur une expérience imposée par les circonstances, nous croyons pouvoir toucher de plus prêt le chiffre de la R.E., du moins dans les limites restreintes à notre cas et à une saison déterminée. Nos conclusions sont tirées d'un résultat global obtenu récemment, pendant une période de 14 mois, dans une station de trutticulture différente de celle qui a servi à nos premiers essais. Cet établịssement, spécialement créé en

(1) Loc. cit., p. 268. 
vue du nourrissage intensif, donnait, en temps normal, de bons rendements en poids, marqués par des accroissements de 30 à $50 \%$ par mois.

Ne trouvant en automne 1940 que des quantités de nourriture très minimes et insuffisantes, même pour une population de Truites considérablement réduite, nous avons procédé, et cela pendant plus d'un an, à des - pesées périodiques, de facon à pouvoir établir la courbe du poids total de notres cheptel et à juger de la possibilité de continuer l'exploitation au ralenti ou de la nécessité de renoncer provisoirement au nourrissage.

Les pesées furent exécutées dans quatre bassins témoins, non cimentés, représentant les diverses catégories de Truites. Celles-ci étaient presque toutes des sujets d'un an et demi. En raison des remaniements, triages et transferts habituels, ces bassins ne furent pas constamment les mêmes; mais la méthode de la fixation approximative du poids global ne fut modifiée à aucun moment.

L'alimentation consistait presque uniquement en abats cuits, si ce n'est ume très faible partie qui put être employée à l'etat cru. Nous aurions voulu y joindre une bonne dose de produits végétaux, de manière à restreindre la part p̀rise à l'entretién par la protéine, conformément à un principe que nous examinerons tout à l'heure, mais cet appoint ne fut réalisé que dans une mesure dérisoire. Les poids de nourriture ayant sẹrvi au calcul des R.P. et des Q.A. sont les poids bruts de la marchandise fournie par les abattoirs; et non ceux, plus fáibles, des rations préparées.

Au début, pendant quelques semaines de meilleur approvisionnement, les pesées révélèrent une progression satisfaisante du poids général ; mais ce moment de prospérité relative fut suivi de nombreux mois entrecoupés de jeûnes.prolongés, et, au bout d'une longue période de 426 jours, l'expérience se termina, en même temps que la liquidation du rayon " nourrissage ", par un résultat peu reluisant. Nous pouvons résumer celui-ci en disant que, pour l'ensemble des quatorze mois compris entre le 25 Septembre' 1940 et le 25 Novembre de l'année suivante, le Q.A. relatif est de 23,3, tandis que le Q.A. absolu, Truites perdues comprises, s'établit à 16,4. Quant à la R.P., elle se monte en moyenne à $3,26 \%$. Ces chiffres anormaux seraient inavouables en d'autres temps; il faut cependant noter et c'est là où nous voulions en venir - que cette faible ration journalière a produit une augmentation de poids, et que, par conséquent, la ration moyenne d'entretien de la durée totale de l'expérience se situe plus bas que $3,26 \%$. 